\title{
Single-nucleon experiments
}

\author{
Alexandre Deur*t \\ Jefferson Lab \\ E-mail: deurpamejlab.org
}

\begin{abstract}
We discuss the Jefferson Lab low momentum transfer data on moments of the nucleon spin structure functions $g_{1}$ and $g_{2}$ and on single charged pion electroproduction off polarized proton and polarized neutron. A wealth of data is now available, while more is being analyzed or expected to be taken in the upcoming years. Given the low momentum transfer selected by the experiments, these data can be compared to calculations from Chiral Perturbation theory, the effective theory of strong force that should describe it at low momentum transfer. The data on various moments and the respective calculations do not consistently agree. In particular, experimental data for higher moments disagree with the calculations. The absence of contribution from the $\Delta$ resonance in the various observables was expected to facilitate the calculations and hence make the theory predictions either more robust or valid over a larger $Q^{2}$ range. Such expectation is verified only for the Bjorken sum, but not for other observables in which the $\Delta$ is suppressed. Preliminary results on pion electroproduction off polarized nucleons are also presented and compared to phenomenological models for which contributions from different resonances are varied. Chiral Perturbation calculations of these observables, while not yet available, would be valuable and, together with these data, would provide an extensive test of the effective theory.
\end{abstract}

6th International Workshop on Chiral Dynamics

July 6-10 2009

Bern, Switzerland

\footnotetext{
*Speaker.

${ }^{\dagger}$ Acknowledgments: This work is supported by the U.S. Department of Energy (DOE). The Jefferson Science Associates (JSA) operates the Thomas Jefferson National Accelerator Facility for the DOE under contract DE-AC0584ER40150.
} 


\section{Introduction and context}

Understanding Nature using the technique of particle scattering is a vast endeavor. As the title of this contribution indicates, we will focus on scattering off the nucleon. This is still a large subject. Consequently, in addition to single nucleon, we will mostly restrict this presentation to single particle detection (i.e. inclusive scattering) from a single type of beam (electron scattering), with single spin directions for the beam and target (i.e doubly polarized scattering) and we will mostly report on results from a single laboratory (Jefferson Lab).

Doubly polarized electron scattering off the nucleon is a powerful tool to understand strong interactions: The polarization provides the most stringent constraints on the theory, while the leptonic probe is the cleanest way to access the structure of the nucleon. Having a single nucleon target removes the difficulties arising from collective effects, such as the EMC effects [1]. Since the nucleon structure is ruled by strong interactions, an understanding of its structure translates to understanding of strong interactions. The gauge theory of strong interactions is Quantum Chromodynamics (QCD). Compared to other interactions, it stands out because it is generally non-perturbative, except for high energy ( $\gg \mathrm{GeV}$ ) reactions. Consequently, the first natural step toward our understanding of strong interactions is to check that QCD is valid where we can analytically solve it, while effective theories (e.g. Chiral Perturbation Theory) or numerical methods (Lattice QCD) need to be developed and tested to cover the lower energy region where QCD is non-perturbative. For final completion of our understanding, one then needs to connect the fundamental and effective theories, just like the empirical rules of chemistry have been fundamentally justified by quantum (atomic) physics, or geometrical optics by electromagnetism. Part of the first step -the validity of perturbative QCD (pQCD) even when spin degrees of freedom are explicit- has been achieved by a first generation of experiments that ran in the 1980's-1990's at facilities such as SLAC, CERN or DESY. Here, we will discuss the experimental program at Jefferson Lab to achieve the other part of the first step -testing effective theories and numerical methods-, and the second step: the connection between $\mathrm{pQCD}$ and the effective descriptions.

\section{Jefferson Lab, the experimental halls and their polarized targets}

Jefferson Lab is an accelerator facility delivering a high quality continuous electron beam with energy up to $6 \mathrm{GeV}$. Beam polarization reaches $85 \%$ and beam current can be up to 200 $\mu \mathrm{A}$. Experiments are carried out in three experimental halls: Halls A, B and C. Hall A [2] and $\mathrm{C}$ contain high resolution spectrometers for high precision experiments with limited phase space coverage. Hall B contains a large acceptance spectrometer [3] for exclusive experiments and/or exploratory measurements over a wide kinematic range. Each hall is home of polarized targets. Hall $\mathrm{A}$ is home of a ${ }^{3} \mathrm{He}$ gaseous target polarized by optical pumping. Polarized ${ }^{3} \mathrm{He}$ acts as an effective polarized neutron target because the dominant nuclear state is the $\mathrm{S}$ state, for which the Pauli principle forces the two proton spins to be anti-aligned. Hence, the single neutron contributes dominantly (about 90\%) to the target polarization. The Hall A target, together with the JLab continuous beam, achieves the largest polarized luminosity in the world $\left(10^{36} \mathrm{~s}^{-1} \mathrm{~cm}^{-2}\right)$. This, with its low dilution from unpolarized materials (typically, about $30 \%$ of the events comes from polarized neutron) and its excellent polarization (60-70\%) allows for high precision experiments. 
The target polarization can be oriented in any direction, including the vertical one. In this talk, we focus on neutron results although data on ${ }^{3} \mathrm{He}$ structure are available as well. This topic is covered by K. Slifer's contribution to this conference. Hall B and Hall C employ solid targets polarized by DNP (Dynamical Nuclear Polarization). The materials most often used are ammonia ${ }^{15} \mathrm{NH}_{3}$ and ${ }^{15} \mathrm{ND}_{3}$. Hence the proton, deuteron and neutron structures are studied in halls $\mathrm{B}$ and $\mathrm{C}$. The targets allow to reach good luminosities $\left(10^{34} \mathrm{~s}^{-1} \mathrm{~cm}^{-2}\right.$ for Hall B and $10^{35} \mathrm{~s}^{-1} \mathrm{~cm}^{-2}$ for Hall $\left.\mathrm{C}\right)$. The somewhat lower luminosity in Hall B is compensated by the large acceptance of the CLAS detector. Solid ammonia targets have relatively high dilution from unpolarized materials (e.g. typically, about $15 \%$ of the events comes from polarized proton) but reach high polarizations $\left(90 \%\right.$ for $\mathrm{NH}_{3}$ and $40 \%$ for $\mathrm{ND}_{3}$ ). The Hall $\mathrm{B}$ target [4] is polarized along the beam direction, while the Hall $\mathrm{C}$ target polarization can be along the beam direction or transverse (in the horizontal plane) to it. Hall $\mathrm{B}$ also is also home of the FROST [5] polarized target, but we will not discuss it since it cannot accommodate electron beams. The former LEGS polarized HD target [6] will also be available soon in Hall B. It is presently being redesigned in order to accommodate electron beams in addition to its original usage with photon beams. If this target does stand electron beams, an important possibility of a transversely polarized target (with low dilution from unpolarized materials) experiments in Hall B will be opened.

\section{Sum rules and the spin structure of the nucleon}

The information on the longitudinal spin structure of the nucleon is contained in the $g_{1}\left(x, Q^{2}\right)$ and $g_{2}\left(x, Q^{2}\right)$ spin structure functions. The kinematic variable $Q^{2}$ is the squared four-momentum transfered from the beam to the target. It fixes the space-time scale at which the nucleon is probed. The other kinematics variable, $x=Q^{2} /(2 M v)$, is the Bjorken scaling variable ( $v$ is the energy transfer from the beam to the target, and $M$ is the nucleon mass). The variable $x$ is interpreted in the parton model as the fraction of nucleon momentum carried by the struck quark. Another kinematics variable that we will use in this talk is $W$ the invariant mass of the recoiling system: $W^{2}=M^{2}+2 M v-Q^{2}=M^{2}+Q^{2}(1 / x-1)$.

Although spin structure functions are the basic observables for nucleon longitudinal spin studies, we will focus on their integrals formed over $x$ and weighted by powers of $x$. Considering these moments is advantageous because of the resulting simplifications. More importantly, such integrals are at the core of the dispersion relation formalism. Dispersion relations relate the integral over the imaginary part of a quantity to its real part. Expressing the imaginary part as a function of the real part using the optical theorem yields sum rules. When additional hypotheses are used, such as a low energy theorem, or the validity of Operator Product Expansion (OPE), the sum rules then relate the integral to a static property of the target, e.g. its anomalous magnetic moment, an electromagnetic polarizability, or its axial charge. If the static property is well known (e.g. the anomalous moment or the axial charge), the verification of the sum rule provides a check of the theory and hypotheses used in the sum rule derivation. When the static property is not known because for instance it is difficult to measure directly (e.g. the generalized electromagnetic polarizabilities), sum rules can be used to access them. In that case, the theoretical framework used to derive the sum rule has to be assumed to be valid. Details on integrals of spin structure functions and sum rules are given e.g. in the review [7]. 
Several spin sum rules exists. We will focus on the Bjorken sum rule [8], the GerasimovDrell-Hearn (GDH) sum rule [9] and spin polarizability sum rules. In this paper, we will consider the $n$-th Cornwall-Norton moments: $\int_{0}^{1} d x g_{1}^{N}\left(x, Q^{2}\right) x^{n}$, with $N$ standing for proton or neutron. We write the first moments as $\Gamma_{1}^{N}\left(Q^{2}\right) \equiv \int_{0}^{1} d x g_{1}^{N}\left(x, Q^{2}\right)$.

\section{The generalized Bjorken and GDH sum rules}

The Bjorken sum rule [8] relates the integral over the isovector part of the first spin structure function, $\int_{0}^{1} d x\left(g_{1}^{p}-g_{1}^{n}\right)$, to the nucleon axial charge $g_{A}$. The original sum rule stands at infinite $Q^{2}$ but has been generalized to finite $Q^{2}$ with the OPE (i.e. pQCD). This relation has been essential for understanding the nucleon spin structure and establishing, via its $Q^{2}$-dependence, that QCD describes the strong force even when spin degrees of freedom are explicit. The Bjorken integral has been measured in polarized deep inelastic lepton scattering (DIS) at SLAC, CERN and DESY [10][15] and at moderate and low $Q^{2}$ at Jefferson Lab (JLab) [16]-[28]. A recent review of these data can be found in [29]. The OPE yields the following expression for the sum rule:

$$
\begin{gathered}
\Gamma_{1}^{p-n}\left(Q^{2}\right) \equiv \int_{0}^{1} d x\left(g_{1}^{p}\left(x, Q^{2}\right)-g_{1}^{n}\left(x, Q^{2}\right)\right)= \\
\frac{g_{A}}{6}\left[1-\frac{\alpha_{s}}{\pi}-3.58 \frac{\alpha_{s}^{2}}{\pi^{2}}-20.21 \frac{\alpha_{s}^{3}}{\pi^{3}}+\ldots\right]+\sum_{i=2}^{\infty} \frac{\mu_{2 i}^{p-n}\left(Q^{2}\right)}{Q^{2 i-2}}
\end{gathered}
$$

where $\alpha_{s}\left(Q^{2}\right)$ is the strong coupling constant. The bracket term (known as the leading twist term) is mildly dependent on $Q^{2}$ due to soft gluon radiations. The summation term contains nonperturbative power corrections (higher twists). These are quark and gluon correlations describing the nucleon structure away from the large $Q^{2}$ (small distances) limit.

The generalized Bjorken sum rule has been derived for small distances. For large distances, at $Q^{2}=0$, one finds the GDH sum rule [9]. For a spin 1/2 target, it reads:

$$
\int_{v_{0}}^{\infty} d v \frac{\sigma_{1 / 2}(v)-\sigma_{3 / 2}(v)}{v}=-\frac{2 \pi^{2} \alpha \kappa^{2}}{M_{t}^{2}}
$$

where $v_{0}$ is the pion photoproduction threshold, $\sigma_{1 / 2}$ and $\sigma_{3 / 2}$ are the helicity dependent photoproduction cross sections when the sum of the photon and target helicities is $1 / 2$ and $3 / 2$, respectively. $\kappa$ is the anomalous magnetic moment of the target and $M_{t}$ its mass. $\alpha$ is the fine structure constant. The GDH sum rule can also be written for target of any spin (e.g. a deuteron target). Replacing the photoproduction cross sections by the electroproduction ones generalizes the left hand side of Eq. 4.2 to any $Q^{2}$, that is, it generalizes the sum. Such generalization depends on the choice of convention for the virtual photon flux, see e.g. ref. [7]. X. Ji and J. Osborne [30] showed that the sum rule itself (i.e. the whole Eq. 4.2) can be generalized as:

$$
\frac{8}{Q^{2}} \int_{0}^{1^{-}} d x g_{1}=S_{1}\left(0, Q^{2}\right)
$$

where $S_{1}\left(v, Q^{2}\right)$ is the spin-dependent Compton amplitude and the $1^{-} \equiv 1-\varepsilon$ integration upper limit excludes the elastic contribution. This generalization of the GDH sum rule makes the connection between the Bjorken and GDH generalized sums evident:

$$
G D H=\frac{Q^{2}}{8} \times \text { Bjorken, }
$$



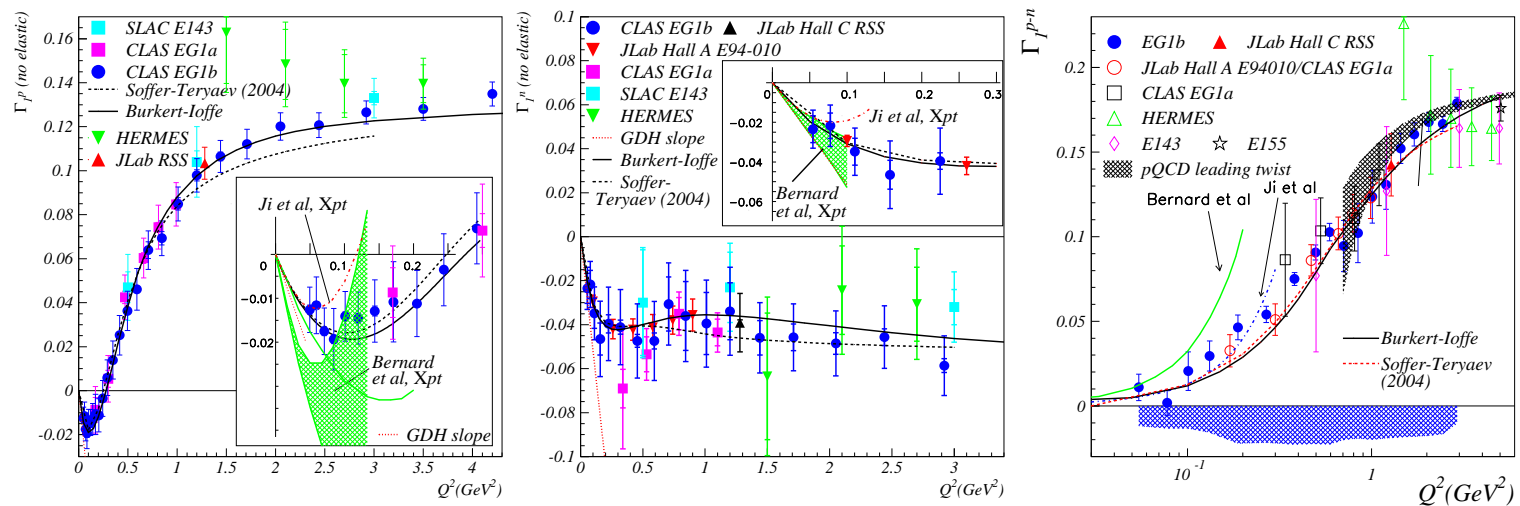

Figure 1: (Color online) Experimental data from SLAC, CERN, DESY and JLab at low and intermediate $Q^{2}$ on $\Gamma_{1}^{p}$ (left), $\Gamma_{1}^{n}$ (center) and $\Gamma_{1}^{p-n}$ (right).

where the elastic contribution to the Bjorken sum, Eq. 4.1, is in this case excluded. The connection between the GDH and Bjorken sum rules makes theories available to compute the moment $\Gamma_{1}$ at any $Q^{2}$. The Bjorken sum rule, evolved with $Q^{2}$ according to $\mathrm{pQCD}$ provides the theoretical prediction at large $Q^{2}$ : Calculation of the spin dependent Compton amplitude $S_{1}\left(v, Q^{2}\right)$ with Lattice QCD (intermediate $Q^{2}$ ) or at low $Q^{2}$ with Chiral Perturbation Theory ( $\chi \mathrm{pT}$, the effective theory of strong force at large distances) yields the theoretical predictions at intermediate and low $Q^{2}$. Thus, we are provided with a convenient observable to understand how the strong force transitions from its description in term of fundamental degrees of freedom (quarks and gluons; small distances) to its description in term of effective degrees of freedom (hadrons; large distances).

\section{Experimental measurements of the first moments}

Results from experimental measurements from SLAC [12], CERN [14], DESY [15] and JLab [16][28] of the first moments $\Gamma_{1}$ are shown in Figure 1. There is an thorough mapping of the moments at intermediate $Q^{2}$ and enough data points at low $Q^{2}$ to start testing $\chi P T$. In this context, the Bjorken sum is especially important because the (p-n) subtraction largely cancels the $\Delta$ resonance contribution which should make the $\chi P T$ calculations significantly more reliable [31]. The comparison between the data at low $Q^{2}$ and $\chi P T$ calculations can be seen on Fig. 1. The calculations are done to next-to-leading order, suing either an explicitly covariant formalism [32] or the Heavy Baryon approximation [33]. The $\chi P T$ calculations do not compute the slope of $\Gamma_{1}$ at $Q^{2}=0$, but takes it from the GDH sum rule prediction since it provides the derivative of $\Gamma_{1}$ at $Q^{2}=0$ (see Eq. 4.4). Consequently, $\chi P T$ calculates the deviation from the slope and this is what one should test. A meaningful comparison is then provided by fitting the lowest $Q^{2}$ data points using the form $\Gamma_{1}^{N}=\frac{\kappa_{N}^{2}}{8 M^{2}} Q^{2}+a Q^{4}+b Q^{6} \ldots$ and compare the obtained value of $a$ to the values calculated from $\chi P T$. Such comparison has been carried out for the proton, deuteron [22] and the Bjorken sum [25] (numerical values are given on Fig. 4). These fits point out the importance of including a $Q^{6}$ term for $Q^{2}<0.1 \mathrm{GeV}^{2}$. The $\chi P T$ calculations agree well with the measurements on the individual nucleons up to $Q^{2} \sim 0.08 \mathrm{GeV}^{2}$ for the Ji. et al calculations. They agree with the measurement 
of the Bjorken sum over a larger $Q^{2}$ span: up to $Q^{2} \sim 0.3 \mathrm{GeV}^{2}$ for the Ji. et al calculations, in accordance with the discussion in [31]. Phenomenological models [34],[35] are in good agreement with the data over the whole $Q^{2}$ range.

\section{Spin polarizability sum rules}

Higher moments of $g_{1}$ and $g_{2}$ are connected by sum rules to electromagnetic polarizabilities. Those characterize the coherent response of the nucleon to photon absorption. They are defined using low-energy theorems in the form of a series expansion in the photon energy. The first term of the series comes from the spatial distribution of charge and current (form factors) while the second term results from the deformation of these distributions induced by the photon (polarizabilities). Hence, polarizabilities are as important as form factors in understanding coherent nucleon structure. Generalized spin polarizabilities describe the response to virtual photons. Using a low energy theorem, the generalized forward spin polarizability $\gamma_{0}$ is defined as:

$$
\Re e\left[g_{T T}\left(v, Q^{2}\right)-g_{T T}^{p o ̂ l e}\left(v, Q^{2}\right)\right]=\left(\frac{2 \alpha}{M^{2}}\right) I_{T T}\left(Q^{2}\right) v+\gamma_{o}\left(Q^{2}\right) v^{3}+O\left(v^{5}\right),
$$

where $g_{T T}$ is the spin-flip doubly-virtual Compton scattering amplitude, and $I_{T T}$ is the coefficient of the $O(v)$ term of the Compton amplitude which can be used to generalize the GDH sum rule to non-zero $Q^{2}$. We have $I_{T T}\left(Q^{2}=0\right)=\kappa / 4$. In practice $\gamma_{0}$ can be obtained from a sum rule which has a derivation akin to that of the GDH sum rule:

$$
\gamma_{0}\left(Q^{2}\right)=\left(\frac{1}{2 \pi^{2}}\right) \int_{v_{0}}^{\infty} \frac{\kappa\left(v, Q^{2}\right)}{v} \frac{\sigma_{T T}\left(v, Q^{2}\right)}{v^{3}} d v
$$

where $2 \sigma_{T T} \equiv \sigma_{1 / 2}-\sigma_{3 / 2}$. We can express the sum rule in terms of the spin structure functions as:

$$
\gamma_{0}\left(Q^{2}\right)=\frac{16 \alpha M^{2}}{Q^{6}} \int_{0}^{x_{0}} d x x^{2}\left(g_{1}-\frac{4 M^{2}}{Q^{2}} x^{2} g_{2}\right) .
$$

Similar relations define the generalized longitudinal-transverse polarizability $\delta_{L T}$ :

$$
\begin{gathered}
\Re e\left[g_{L T}\left(v, Q^{2}\right)-g_{L T}^{\text {pole }}\left(v, Q^{2}\right)\right]=\left(\frac{2 \alpha}{M^{2}}\right) Q I_{L T}\left(Q^{2}\right)+Q \delta_{L T}\left(Q^{2}\right) v^{2}+O\left(v^{4}\right), \\
\delta_{L T}\left(Q^{2}\right)=\left(\frac{1}{2 \pi^{2}}\right) \int_{v_{0}}^{\infty} \frac{\kappa\left(v, Q^{2}\right)}{v} \frac{\sigma_{L T}\left(v, Q^{2}\right)}{Q v^{2}} d v \\
\delta_{L T}\left(Q^{2}\right)=\frac{16 \alpha M^{2}}{Q^{6}} \int_{0}^{x_{0}} d x x^{2}\left(g_{1}+g_{2}\right) .
\end{gathered}
$$

where $g_{L T}$ is the longitudinal-transverse interference amplitude, $I_{L T}$ is the coefficient of the $O(v)$ term of the Compton amplitude, and $\sigma_{L T}$ is the longitudinal-transverse interference partial crosssection. Details on the derivations of Eqs. 6.1-6.6 can be found in [7]. Higher moments are advantageous because, thanks to their extra $x^{n}$ weighting, they are essentially free of the uncertainty associated with the low- $x$ extrapolation of the data: Reaching $x \rightarrow 0$ would require an infinite beam 

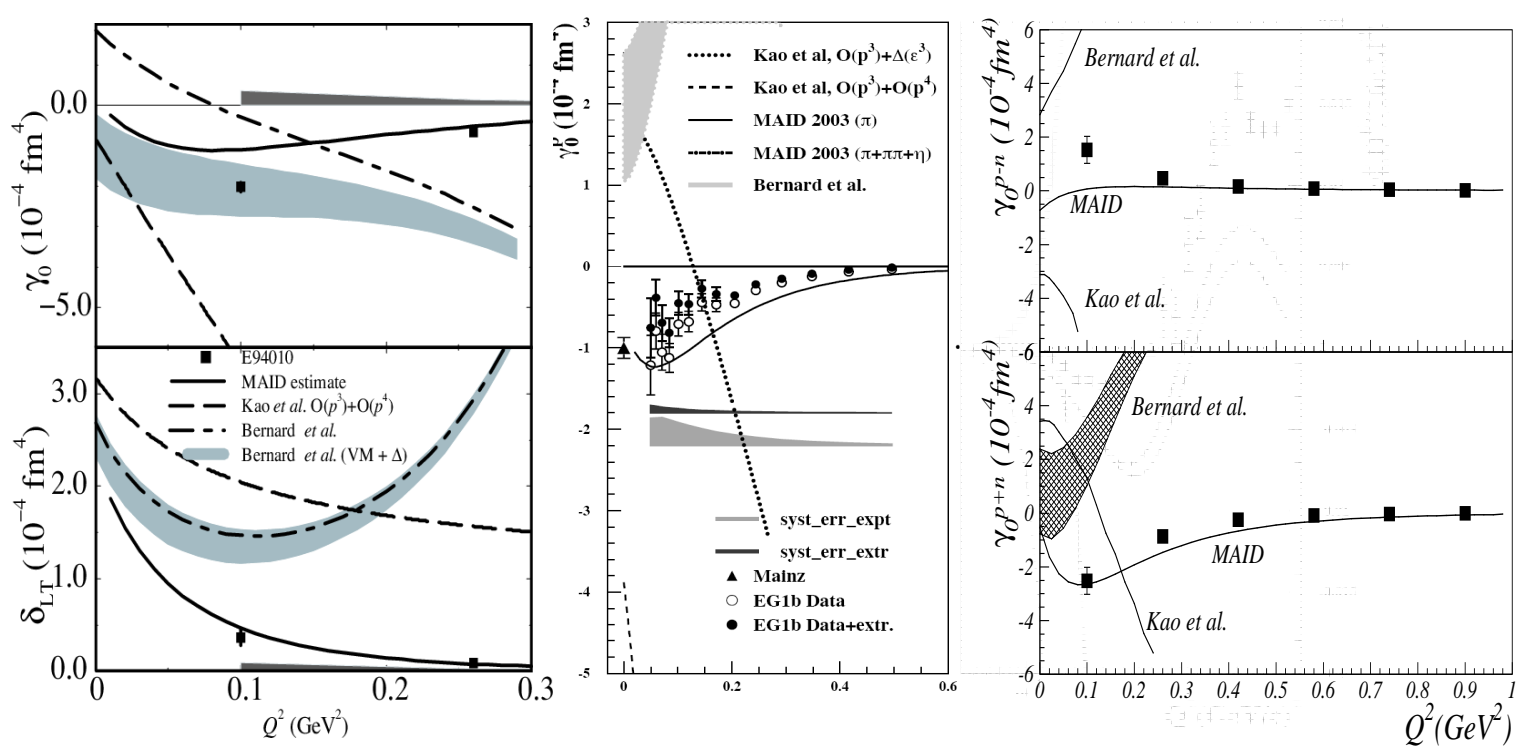

Figure 2: Experimental data at low $Q^{2}$ on generalized spin polarizabilities. Results on neutron (JLab Hall A experiment E94010 [20]) are shown on the left (top: $\gamma_{0}^{n}$, bottom: $\delta_{L T}^{n}$ ). Results on the proton (JLab CLAS experiment EG1b [22]) $\gamma_{0}^{p}$ are shown in the central plot. The isospin decomposition of $\gamma_{0}$ (E94010+EG1b [25]) is shown on the right (top: $\gamma_{0}^{p-n}$, bottom $\gamma_{0}^{p+n}$.

energy and hence, no data exist below a given value of $x$. Eqs. 6.3 and 6.6 are examples of usage of sum rules to measure observables that are otherwise hard to access.

In the case of the transverse-longitudinal polarizability $\delta_{L T}$, the $\Delta$ contribution is suppressed at low $Q^{2}$ because the $\mathrm{N}-\Delta$ transition is mostly transverse, which makes the contribution of the $\Delta$ to the longitudinal-transverse (LT) interference term very small. Thus, similarly to the Bjorken sum (but with a different reason for the $\Delta$ suppression), $\delta_{L T}$ should also provide a robust observable to compute within the $\chi P T$ framework. Furthermore, there is the possibility that the suppression of the $\Delta$ in isovector $(p-n)$ quantities such as the Bjorken sum holds only to the first order $\Delta$ contribution $\gamma^{*} N \rightarrow N \pi$ but not for the second order $\Delta$ contribution $\gamma^{*} N \rightarrow \Delta \pi$. In contrast the general argument explaining the $\Delta$ suppression for $\delta_{L T}$ should hold at all orders. If so, $\delta_{L T}$ would provide an even more robust observable than the Bjorken sum to compute in $\chi P T$. Finally, as for the Bjorken sum, the isovector part of $\gamma_{0}, \gamma_{0}^{p}-\gamma_{0}^{n}$, should offer similar advantages (at least for the $\gamma^{*} N \rightarrow N \pi$ contribution) for checking the calculation techniques of $\chi P T$.

The low $Q^{2}$ data on forward spin polarizabilities, from Hall A E94010 and CLAS EG1b, are shown on Fig. 2. There is no agreement between the data and the $\chi P T$ calculations (except possibly for the lowest $Q^{2}$ point of $\gamma_{0}^{n}$ that agrees with the explicitly covariant calculation of Bernard et al). Such disagreement is surprising because the lowest $Q^{2}$ points should be well into the validity domains of $\chi P T$. It is even more surprising for $\gamma_{0}^{p-n}$ because of the $\Delta$ suppression for this quantity, and $a$ fortiori for the discrepancy with $\delta_{L T}^{n}$ for which we are sure that the $\Delta$ suppression is valid at all orders. This reveals that the $\Delta$ alone is not the only cause of the lack of agreement between data and theory, and including the $\Delta$ contribution $\gamma^{*} N \rightarrow \Delta \pi$ in the calculations may not be the only 


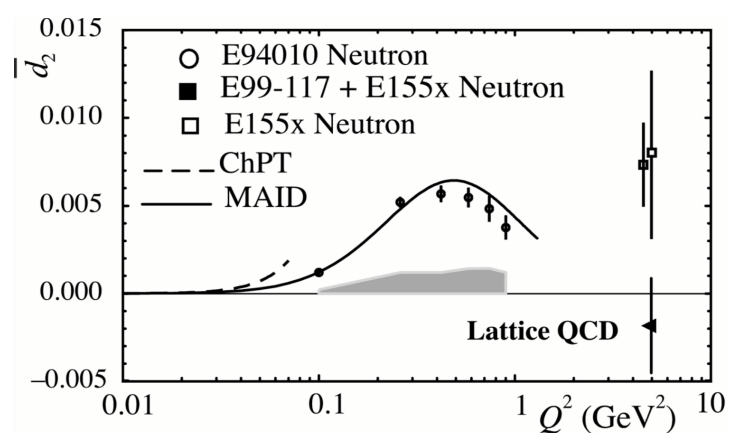

Figure 3: Results on $d_{2}^{n}$ from JLab experiments E94010, E99-117 and SLAC experiment E155x. The plain line indicates the MAID model expectation and the dashed line is for the $\chi P T$ calculations.

challenge facing $\chi P T$ calculations. In contrast, the MAID model [36] is in good agreement with the data, with the notable exception of the $Q^{2}=0.1 \mathrm{GeV}^{2}$ point for $\gamma_{0}^{n}$. Compared to first moments, a better agreement with higher moments is expected for MAID since it mostly includes one pion production reactions and these high- $x$ reactions dominantly contribute to the higher moments.

\section{Higher moment $d_{2}$}

Another higher moment of interest is $d_{2}$. It can be expressed as the third moment of the twist-3 part of $g_{2}$ :

$$
d_{2}=3 \int_{0}^{1} d x x^{2}\left(g_{2}\left(x, Q^{2}\right)-g_{2}^{W W}\left(x, Q^{2}\right)\right)
$$

where $g_{2}^{W W}$ is the pure twist-2 part of $g_{2}$, first isolated by Wandzura and Wilczek [37]. It is a function of the leading twist expression of $g_{1}$ :

$$
g_{2}^{W W}\left(x, Q^{2}\right)=-g_{1}^{L T}\left(x, Q^{2}\right)+\int_{x}^{1} d y\left(g_{1}^{L T}\left(y, Q^{2}\right) / y\right) .
$$

Hence, at large $Q^{2}, d_{2}$ can be cleanly interpreted as a twist-3 quantity (although, see [38]). Its expression in term of $g_{1}$ and $g_{2}$ is:

$$
d_{2}=\int_{0}^{1} d x x^{2}\left(2 g_{1}\left(x, Q^{2}\right)+3 g_{2}\left(x, Q^{2}\right)\right)
$$

At intermediate $Q^{2}$ other higher twists contribute, while this pQCD interpretation in term of parton breaks down at low $Q^{2}$. We can recombine the data or the calculations on $\gamma_{0}$ and $\delta_{L T}$ discussed in the previous section to form $d_{2}$. Hence, there is no new information here regarding the data or the calculations and this recombination only recasts them in a quantity that we can cleanly interpret as a twist-3 element at large $Q^{2}$. At present, only neutron data from Hall A are available to form $d_{2}$ because its measurement requires a transverse target. Fig. 3 displays the results on $d_{2}^{n}$ from JLab experiment E94010 [19], and combined JLab E99-117 [39]/SLAC E155x [13] experiments [39]. The dashed line indicates one of the $\chi P T$ prediction (the two calculations [32],[33] yield very similar results). There is no agreement between data and the $\chi P T$ calculations. In contrast, there is again a good agreement with the MAID model expectation (indicated by the plain line). 


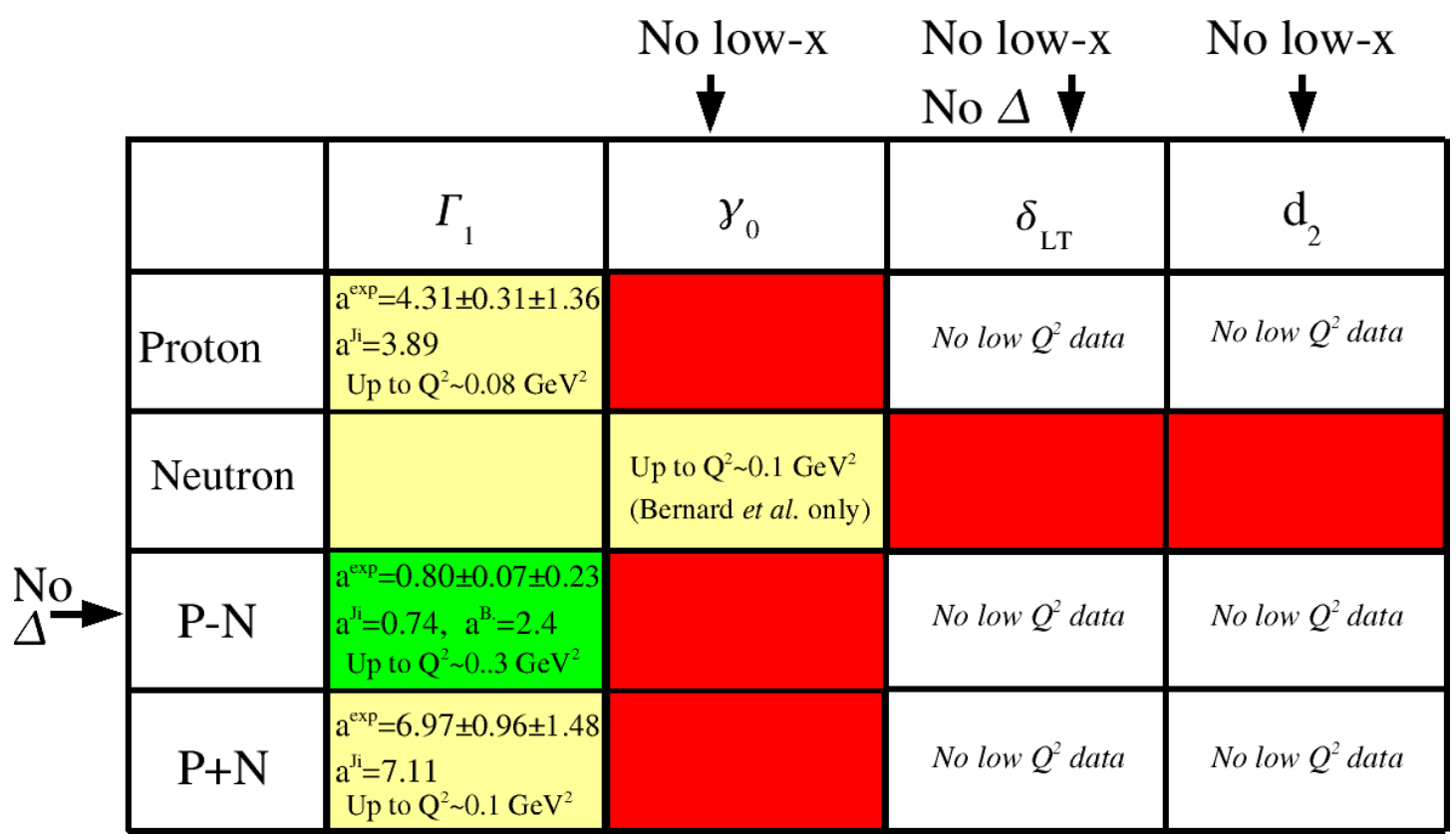

Figure 4: (Color online) Summary the comparison between $\chi P T$ calculations and data. The green indicates a good match within the region in which we expect the chiral perturbation series to be reliable, the yellow an agreement over a shorter $Q^{2}$ range, and the red a mismatch.

\section{Summary of the $\chi P T$ checks and perspectives}

Figure 4 summarizes the comparison between $\chi P T$ calculations and data. It is hard to find a pattern on this summary table: It was expected that the rows or columns labelled "no $\Delta$ " or "no low$\mathrm{x}$ " should have provided robust $\chi P T$ calculations, and a fortiori for the intercept between the two labels $\left(\delta_{L T}, \gamma_{0}^{p-n}\right.$ and $\left.d_{2}^{p-n}\right)$. Hence, the best agreement was expected for these rows or columns. Furthermore, the fact that the $\Delta$ suppression is sure to be valid at all orders for $\delta_{L T}$ singles out this quantity as possibly the most robust calculation. Such pattern is not seen. This cannot be due to the fact that, for first moments, the leading term is given by sum rules instead of being computed by $\chi P T$ as is done for the higher moments. This is not the reason because for the first moments the comparison is done on the second order term obtained from a fit to the data. The table emphasizes that more work is needed on the theoretical side (red boxes) as well as the experimental side (white boxes) for a better understanding of this problem.

The data discussed so far were taken at JLab for experiments focused on covering the intermediate $Q^{2}$ range $[16,25]$. A new generation of experiments, E97110 in Hall A [40], and EG4 in Hall B [41], that were especially dedicated to push such measurements to lower $Q^{2}$ and higher precision, has provided new data that are being analyzed. During this conference, preliminary results on E97110 were presented by V. Sulkosky, while an update on EG4 was provided by S. Phillips. In addition, a new experiment to measure $\delta_{L T}^{p}$ in Hall A at low $Q^{2}$ has been approved by the JLab PAC [42], while the frozen spin HD target recently arrived at JLab from BNL is opening new possibilities of measurements with CLAS using transversely polarized protons or deuterons. 


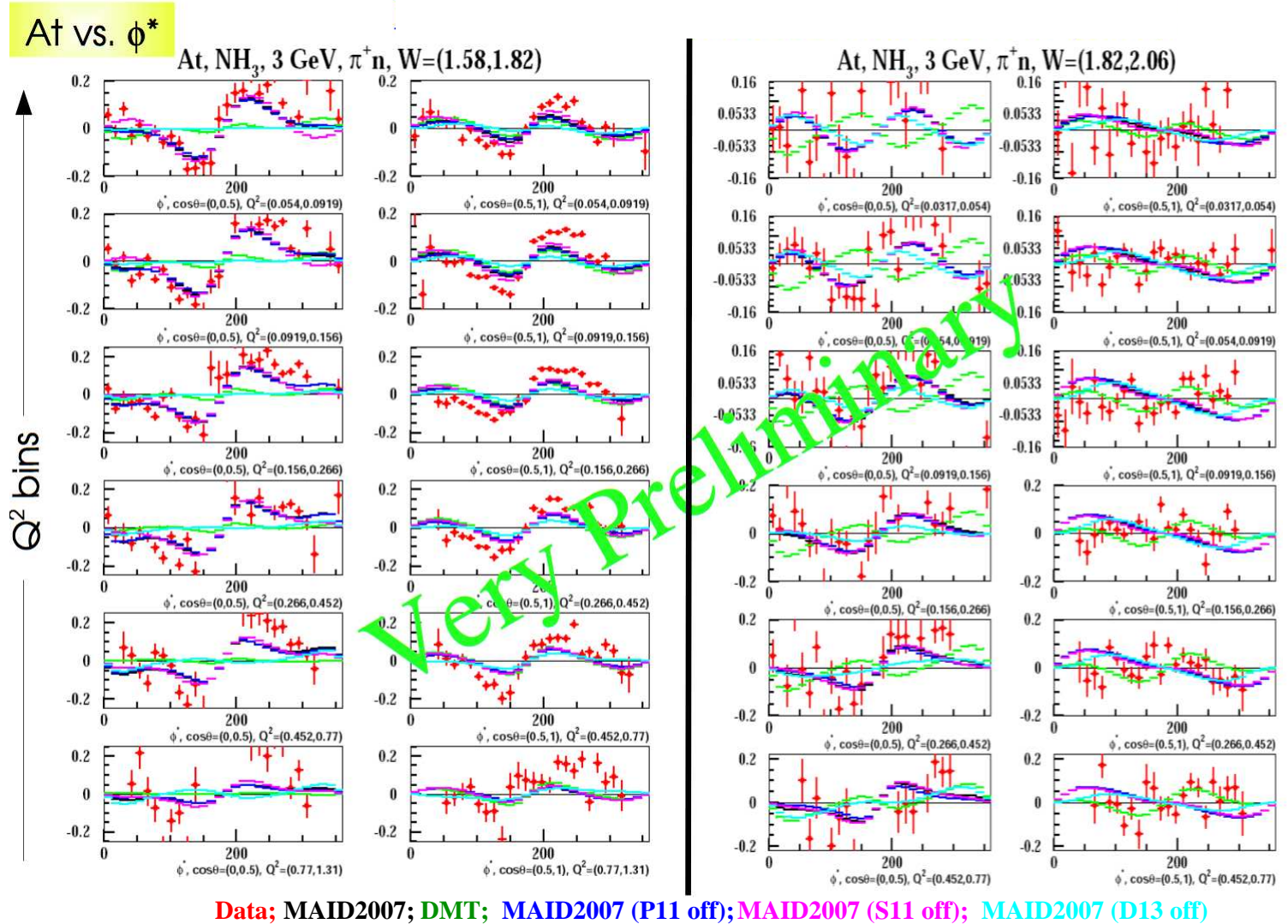

Figure 5: The preliminary $\pi^{+}$single spin asymmetry $A_{t}$ from the 3 GeV CLAS EG4 data [43].

\section{Doubly polarized pion electroproduction off proton and deuteron in the $\chi P T$ domain}

The main goal of the CLAS EG4 experiment was to gather doubly-polarized inclusive data at low $Q^{2}$. However, a large quantity of (mostly single) pion electroproduction events is present. Reactions $\vec{e} \vec{p} \rightarrow e^{\prime} \pi^{+} n$ and $\vec{e} \vec{p} \rightarrow e^{\prime} \pi^{0} p$ are available from the $\mathrm{NH}_{3}$ target and $\vec{e} \vec{n} \rightarrow e^{\prime} \pi^{-} p$ and $\vec{e} \vec{n} \rightarrow e^{\prime} \pi^{0} n$ are available from the $\mathrm{ND}_{3}$ target. Of the three independent asymmetries: $A_{e}$ (single beam asymmetry), $A_{t}$ (single target) asymmetry and $A_{e t}$ (double beam-target asymmetry), only $A_{t}$ and $A_{e t}$ is being analyzed since $A_{e}$ can be accessed more efficiently with unpolarized targets. Data are available from the four EG4 beam energies (3.0, 2.0, 1.3 and $1.1 \mathrm{GeV})$ and, by the design of the experiment, these data belong to the low $Q^{2}$ domain where $\chi P T$ can be applied for low enough $W$. Fig. 5 displays $A_{t}$ for $e \vec{p} \rightarrow e^{\prime} \pi^{+} n$ from the $3 \mathrm{GeV}$ data in function of $\phi^{*}$, the angle between the scattering plane and the reaction plane. Each plot corresponds to different $Q^{2}$ and $W$ range. Since the correction for the dilution from the unpolarized components of the ammonia target is not applied yet to the data, the results from phenomenological models MAID and DMT (DubnaMainz-Taiwan collaboration) [44] are scaled down by a factor 0.2 , the approximately expected dilution of the data. No acceptance corrections are applied yet in this analysis and those might modify the features seen on Fig. 5. Nevertheless, the models and preliminary data are qualitatively 
similar. The important information from these plots, that presents only a fraction of the analyzed data, is the quality of the data accuracy, the extensive kinematic range on which data are available, and the fact that part of the data are taken in the applicability domain of $\chi P T$. No $\chi P T$ calculations are available yet for such reactions. The wealth and quality of data warrant thorough tests of $\chi P T$ if such calculations become available.

\section{Summary and perspectives}

We discussed the Jefferson Lab data on moments of spin structure functions at large distances and compared them to $\chi P T$, the effective theory of strong force that should describe it at large distances. The data and calculations do not consistently agree. In particular, the better agreement expected for observables in which the $\Delta$ resonance is suppressed is seen only for the Bjorken sum, but not for $\gamma_{0}^{p}-\gamma_{0}^{n}$ nor for $\delta_{L T}^{n}$ even if for this latest quantity we are sure that the $\Delta$ is suppressed at all orders, while this is not certain for the isovector $(p-n)$ quantities. Apparently, the $\Delta$ cannot explain single-handedly the discrepancy between data and calculations. The new generation of experiments that gathered data at lower $Q^{2}$, E97110 and EG4 might help shed light on this problem. In addition, data from transversely polarized targets are likely to be crucial to solve the puzzle. These data should be provided by the new E08027 experiment to measure $\delta_{L T}^{p}$ in Hall A at low $Q^{2}$, and possibly by electron scattering experiments using the Hall B frozen spin HD target with CLAS. The analysis of the large amount of doubly polarized pion electroproduction data in the low $Q^{2}$ domain from the EG4 experiment is well advanced. The preliminary results are being compared to phenomenological models. $\chi P T$ predictions are not available so far for these observables but would be very valuable.

\section{References}

[1] J.J. Aubert et al., Phys. Lett. B 123, 275 (1983); For a review, see e.g. D. F. Geesaman, K. Saito and A.W. Thomas, Annu. Rev. Nucl. Part. Sci. 45337 (1995)

[2] J. Alcorn et al., Nuc. Inst. Meth A522 294 (2004)

[3] B. Mecking, et al., Nucl. Inst. and Meth. 503/3, 513 (2003).

[4] C. D. Keith, et al., Nucl. Inst. and Meth. 501, 327 (2003).

[5] C. D. Keith, AIP Conf. Proc. 1149886 (2009).

[6] S. Hoblit, A.M. Sandorfi, et al. Phys. Rev. Lett. 102, 172002 (2009).

[7] J.-P. Chen, A. Deur, Z.-E. Meziani; Mod. Phys. Lett. A 20, 2745 (2005).

[8] J. D. Bjorken, Phys. Rev. 148, 1467 (1966); D 1, 465 (1970); Phys. Rev. D 1, 1376 (1970).

[9] S. D. Drell and A. C. Hearn, Phys. Rev. Lett. 16, 908 (1966). S. Gerasimov, Sov. J. Nucl. Phys. 2, 430 (1966).

[10] P. L. Anthony et al., Phys. Rev. Lett. 71, 959 (1993).

[11] K. Abe et al., Phys. Rev. Lett. 74, 346 (1995); 75, 25 (1995); 76, 587 (1996); Phys. Lett. B 364, 61 (1995); Phys. Rev. D 58, 112003 (1998).

[12] K. Abe et al., Phys. Rev. Lett. 79, 26 (1997). 
[13] P. L. Anthony, et al., Phys. Lett. B 458, 529 (1999); B 463, 339 (1999); B 493, 19 (2000); B 553, 18 (2003).

[14] D. Adeva et al., Phys. Rev. D 58, 112001 (1998).

[15] K. Ackerstaff, et al., Phys. Lett. B 404, 383 (1997); B 444, 531 (1998); A. Airapetian, et al., Phys. Lett. B 442, 484 (1998); Phys. Rev. Lett. 90, 092002 (2003); Eur. Phys. J. C 26, 527 (2003); Phys. Rev. D 75, 012007 (2007).

[16] R. Fatemi et al., Phys. Rev. Lett. 91, 222002 (2003).

[17] J. Yun et al., Phys. Rev. C 67, 055204 (2003).

[18] M. Amarian et al., Phys. Rev. Lett. 89, 242301 (2002).

[19] M. Amarian et al., Phys. Rev. Lett. 92, 022301 (2004).

[20] M. Amarian et al., Phys. Rev. Lett. 93, 152301 (2004).

[21] V. Dharmawardane et al., Phys. Lett. B 641, 11 (2006).

[22] Y. Prok et al., Phys. Lett. B 67212 (2009).

[23] P. Bosted et al., Phys. Rev. C 75035203 (2007).

[24] A. Deur et al., Phys. Rev. Lett. 93, 212001-1 (2004).

[25] A. Deur et al., Phys. Rev. D 78032001 (2008).

[26] F. R. Wesselmann et al, Phys. Rev. Lett. 98132003 (2007)

[27] P. Solvignon et al, Phys. Rev. Lett. 101182502 (2008)

[28] K. Slifer, arXiv:0812.0031 (2009).

[29] S. E. Kuhn, J.-P. Chen, E. Leader, Prog. Part. Nucl. Phys. 631 (2009)

[30] X. Ji et J. Osborne, J. of Phys. G 27, 127 (2001).

[31] V. D. Burkert, Phys. Rev. D 63, 097904 (2001).

[32] V. Bernard, T. R. Hemmert and Ulf-G. Meissner, Phys. Rev. D 67, 076008 (2003).

[33] X. Ji, C. W. Kao and J. Osborne, Phys. Lett. B 472, 1 (2000).

[34] V. D. Burkert and B. L. Ioffe, Phys. Lett. B 296, 223 (1992); J. Exp. Theor. Phys. 78, 619 (1994).

[35] J. Soffer and O. V. Teryaev, Phys. Lett. B 545, 323 (2002); Phys. Rev. D 70116004 (2004).

[36] D. Drechsel, S. Kamalov and L. Tiator. Nucl. Phys. A 645, 145 (1999).

[37] S. Wandzura and F. Wilczek, Phys. Lett. B 72, 195 (1977).

[38] A. Accardi, A. Bacchetta, W. Melnitchouk, M. Schlegel, arXiv:0907.2942. See also AIP Conf. Proc. 115535 (2009).

[39] X. Zheng et al. Phys. Rev. Lett. 92, 012004 (2004); Phys. Rev. C 70, 065207 (2004).

[40] J.P. Chen, A. Deur and F. Garibaldi, JLab experiment E97-110.

[41] M. Battaglieri, A. Deur, R. De Vita and M. Ripani, JLab experiment E03-006. A. Deur, G. Dodge and K. Slifer, JLab PR05-111.

[42] K. Slifer, A. Camsonne and J-P. Chen, JLab experiment E08027.

[43] X. Zheng, AIP Conf. Proc. 1155135 (2009).

[44] S. Kamalov and S. Yang, Phys. Rev. Lett. 834494 (1999); S. Kamalov, S. Yang, D. Drechsel, O. Hanstein, L. Tiator, Phys. Rev. C 64032201 (2001). 